\title{
Cardiovascular Physiotherapy on Respiratory Sinus Arrhythmia of Patients Undergoing Coronary Artery Bypass Grafting
}

Bianca Lopes Silva1,2, PT; Roberto Ribeiro da Silva ${ }^{1,3}$, PT; Hugo Valverde Reis ${ }^{1,3}$, PT; Ana Carolina Accosio Rodriguez ${ }^{1,3}$, PT; Priscila Souza e Souza ${ }^{1}$, PT; Isabela de Andrade ${ }^{1,3}$, PT; Leonardo Fonseca ${ }^{4}$, PT; Solange Guizillini ${ }^{5}$, PhD; Michel Silva Reis ${ }^{1,2,3}, \mathrm{PhD}$

DOI: $10.21470 / 1678-9741-2020-0276$

\begin{abstract}
Introduction: Patients in the postoperative period of coronary artery bypass grafting (CABG) present respiratory and autonomic dysfunctions. In this sense, cardiovascular physiotherapy has been offered as an indispensable differential for the improvement of the prognosis of this population. Heart rate variability is a simple, noninvasive method to analyze autonomic modulation, as well as the accentuation maneuver of respiratory sinus arrhythmia,
\end{abstract}

which demonstrates the parasympathetic autonomic control over the heart. Five patients undergoing cardiac surgery performed a protocol of cardiovascular physiotherapy in the postoperative period and had their data referring to the preoperative period, the 1st and 4th postoperative days analyzed.

Keywords: Heart Rate Variability. Respiratory Sinus Arrhythmia Maneuver. Cardiovascular Physiotherapy. Coronary Artery Bypass Grafting.

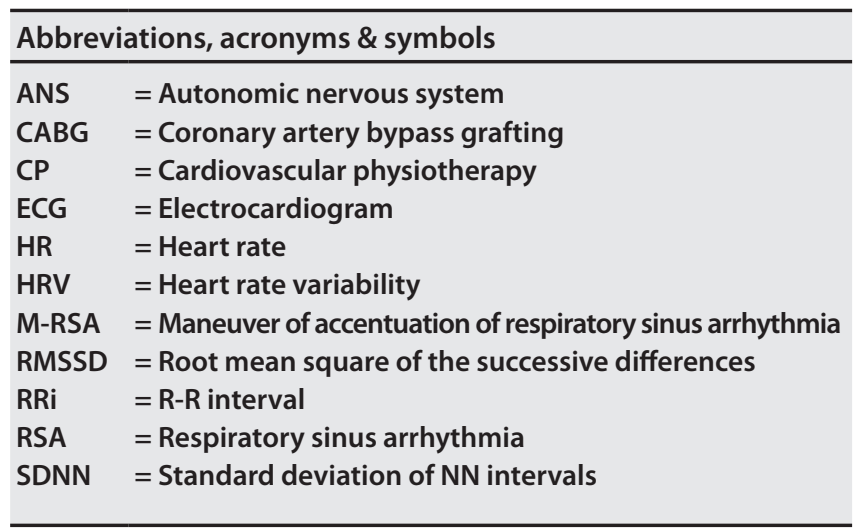

\section{INTRODUCTION}

Cardiovascular physiotherapy (CP) is an integral part of the care of patients in the postoperative period of coronary artery bypass grafting (CABG), since it contributes to a better prognosis regarding the recovery of autonomic and respiratory disorders present in the period. In this context, the autonomic heart rate (HR) modulation can be evaluated in a non-invasive way, based on the analysis of heart rate variability (HRV) and $H R$ response ${ }^{[1,2]}$ in autonomic function tests, as in the maneuver of accentuation of respiratory sinus arrhythmia (M-RSA) ${ }^{[3,4]}$

Respiratory sinus arrhythmia (RSA) is a physiological event characterized by HR oscillations associated with the respiratory cycle ${ }^{[5]}$. Such phenomenon seems to occur by an inhibition of parasympathetic activity during inspiration, with M-RSA being a tool to evaluate this autonomic control. Reduction in HRV and RSA may be associated with abnormal and insufficient autonomic nervous system (ANS) adaptability over cardiac autonomic control of patients after CABG and have an influence on their prognosis ${ }^{[1,6]}$. Pulmonary dysfunction, associated with respiratory complications, such as the need to remain with invasive or non-invasive ventilatory support for longer than scheduled, atelectasis and hypoxemia, increase the rate of morbidity and mortality in this period ${ }^{[7,8]}$.
${ }^{1}$ Faculty of Physiotherapy, Research Group in Cardiorespiratory Evaluation and Rehabilitation (GECARE), Federal University of Rio de Janeiro (UFRJ), Rio de Janeiro, RJ, Brazil.

${ }^{2}$ Graduate Program in Physical Education, Federal University of Rio de Janeiro (UFRJ), Rio de Janeiro, RJ, Brazil.

${ }^{3}$ Graduate Program in Cardiology, Federal University of Rio de Janeiro (UFRJ), Rio de Janeiro, RJ, Brazil.

${ }^{4}$ Federal Hospital of State Public Servants, Rio de Janeiro, RJ, Brazil.

${ }^{5}$ Federal University of São Paulo (UNIFESP), São Paulo, SP, Brazil.

This study was carried out at the Federal University of Rio de Janeiro (UFRJ), Rio de Janeiro, RJ, Brazil.
Article received on June $3^{\text {rd }}, 2020$ Article accepted on August 17 1 th 2020. 
Currently, few studies contemplate the HRV analysis in the postoperative period of CABG with the application of an exercisebased CP protocol, and none of them provides complementary data on the behavior of RSA in this period. However, the analysis of this variable brings information about the integrity of parasympathetic modulation and how the ventilatory mechanics can alter this modulation ${ }^{[9,10]}$. In view of this, our objective was to evaluate the effect of CP on cardiac autonomic modulation during the postoperative period of cardiac surgery.

\section{COMMENTS}

Five patients undergoing CABG were admitted to the Coronary Care Unit of the Federal Hospital of State Public Servants of Rio de Janeiro. They performed a CP protocol in the postoperative period and had their data referring to the preoperative period, the $1^{\text {st }}$ and $4^{\text {th }}$ postoperative days analyzed. This study was approved by the Ethics Committee of Federal Hospital of State Public Servants of Rio de Janeiro (CAEE: 39101114.2.0000.5257). Initially, the patients underwent two types of daily evaluations: (i) Clinical cardiological evaluation performed daily by the unit's physician. This assessment consisted of routine tests to characterize the clinical evolution, arterial blood gas analysis, laboratory tests (blood count, biochemistry, electrolytes) and electrocardiogram; (ii) Physiotherapy evaluation: consisting of anamnesis and physical examination, to investigate the history and current illness, as well as life and eating habits. The evaluations were performed daily, always at the same time of day, considering circadian variations, to define their progression or not in the experimental protocol.

CP consisted of: (i) $1^{\text {st }}$ postoperative day - Head of bed elevated 450: breathing exercises (4 min) and active-assisted exercises of upper and lower limbs - 3 sets of 10 repetitions; (ii) $2^{\text {nd }}$ postoperative day: Patient seated at $90^{\circ}$ : breathing exercises ( $4 \mathrm{~min}$ ) and active-free exercises of upper and lower limbs (2 sets of 15 repetitions). Orthostatic posture: stationary walking (5 min or limited by symptoms); $3^{\text {rd }}$ postoperative day: Seated patient: breathing exercises and repetition of stage 2 with 3 sets of 15 repetitions. Ambulation in the corridor ( $5 \mathrm{~min}) ; 4^{\text {th }}$ postoperative day: Seated patient: repetition of stage 3. Ambulation in the corridor (10 $\mathrm{min}) ; 5^{\text {th }}$ postoperative day: Patient in orthostatic position: repeat stage 3. Ambulation in the corridor (10 min) and up and down stairs (4 steps).

HR and R-R intervals (RRi) were recorded beat by beat using a heart rate monitor (Polar RS800). While using this heart rate monitor, data was transmitted simultaneously to the watch where it was stored. The collection was performed at rest in the supine position for 10 minutes. The same heart rate monitor was used during the performance of the M-RSA, when the patient was instructed, by verbal and tactile command (abdominal stimulation), to perform deep and slow nasal inspirations and oral expirations, varying the pulmonary volume from total lung capacity to residual volume, according to the existing protoco|[5]. HRV data were analized using Kubius HRV 2.0 application for Windows. Initially, from the visual inspection, the 5-minute stretches with the highest stability of the resting ECG RRi tracing were selected. Time domain analysis was performed by means of HR, mean RRi, standard deviation of NN intervals (SDNN) and root mean square of the successive differences (RMSSD) indexes ${ }^{[1]}$. Then, through a specific routine developed in the MATLAB application, the following HR and RRi indexes obtained from M-RSA were calculated: expiration/inspiration ratio (E/I) and inspiration-expiration difference $(\Delta \mathrm{IE})^{[3]}$. All patients who participated in the study started the protocol from the $1^{\text {st }}$ postoperative day after orotracheal extubation and with medical consent and performed the activities once a day for at least five days of hospitalization according to Table $1^{[10]}$.

Table 1. Anthropometric data and clinical characteristics of the patients.

\begin{tabular}{l|c}
\hline Variables & Patients $(\mathbf{n}=\mathbf{5})$ \\
\hline Age (years) & $66.4 \pm 10$ \\
\hline Gender $(M / F)$ & $03 / 02$ \\
\hline Height $(\mathrm{m})$ & $1.6 \pm 1$ \\
\hline Body mass $(\mathrm{kg})$ & $76.1 \pm 10$ \\
\hline BMl $\left(\mathrm{kg} / \mathrm{m}^{2}\right)$ & $29.5 \pm 6$ \\
\hline
\end{tabular}

\section{Clinical features}

\begin{tabular}{l|c}
\hline Resting HR $(\mathrm{bpm})$ & $63.6 \pm 10$ \\
\hline Resting SBP $(\mathrm{mmHg})$ & $110 \pm 12$ \\
\hline Resting DBP $(\mathrm{mmHg})$ & $66.8 \pm 6$ \\
\hline $\mathrm{SpO}_{2}$ & $96.8 \pm 1$ \\
\hline Lesion (n/\%) & $2 / 40$ \\
\hline Bivascular & $3 / 60$ \\
\hline Multivascular
\end{tabular}

\section{Risk factors (n/\%)}

\begin{tabular}{l|c}
\hline SAH & $5 / 100$ \\
\hline DM & $1 / 20$ \\
\hline Dyslipidemia & $3 / 60$ \\
\hline Positive FH & $4 / 80$
\end{tabular}

\section{Surgical data}

\begin{tabular}{l|c}
\hline CPB (n/\%) & $2 / 40$ \\
\hline CPB (min) & $35.8 \pm 50$ \\
\hline MV (min) & $178 \pm 273$ \\
\hline
\end{tabular}

\section{Drug treatment $(\mathrm{n} / \%)$}

\begin{tabular}{l|l}
\hline Beta blocker & $4 / 80$ \\
\hline ACEi & $3 / 60$ \\
\hline Diuretics & $4 / 80$ \\
\hline
\end{tabular}

Values expressed as mean \pm standard deviation. $\mathrm{ACE}$ =angiotensinconverting-enzyme inhibitors; $B M l=b o d y$ mass index; $\mathrm{CPB}=$ cardiopulmonary bypass; $\mathrm{DBP}=$ diastolic blood pressure; $\mathrm{DM}=$ diabetes mellitus; $\mathrm{FH}=$ family history; $\mathrm{HR}=$ heart rate; $\mathrm{HT}$ : hypertension; $\mathrm{MV}=$ mechanical ventilation; $\mathrm{SBP}=$ systolic blood pressure; $\mathrm{SpO}_{2}=$ peripheral oxygen saturation 
The data were analyzed by descriptive statistics organized in Sigmaplot (version 11 for Windows). The results were presented as mean and standard deviation. The anthropometric data and general characteristics of the individuals were shown in Table 1. HRV and M-RSA values were shown in Figure 1.

The main findings of this study are that HRV indexes of postCABG patients undergoing CP showed improvement in the index related to parasympathetic modulation (RMSSD). Additionally, on the $4^{\text {th }}$ postoperative day, most patients had values like those in the preoperative period. Interestingly, during the M-RSA, the values that represent the amplitude of the RSA showed a reduction, improving in the $4^{\text {th }}$ postoperative day. It is already consolidated in the literature that, immediately after CABG, the autonomic dysfunction is present and this dysfunction is related to a greater number of cardiovascular complications, worse prognosis and higher mortality ${ }^{[6,7,9,10]}$.

Soares et al. ${ }^{[11]}$ (2005) showed that cardiac autonomic modulation worsens substantially in the first six postoperative days and the reversibility of this worsening occurs only 60 days after CABG. However, in our study, for four days a behavior of return to the basal values could already be observed in some important variables such as HR and SDNN index. Some studies ${ }^{[11,12]}$ have already demonstrated that physical exercise during the postoperative period of CABG has several benefits in autonomic modulation.
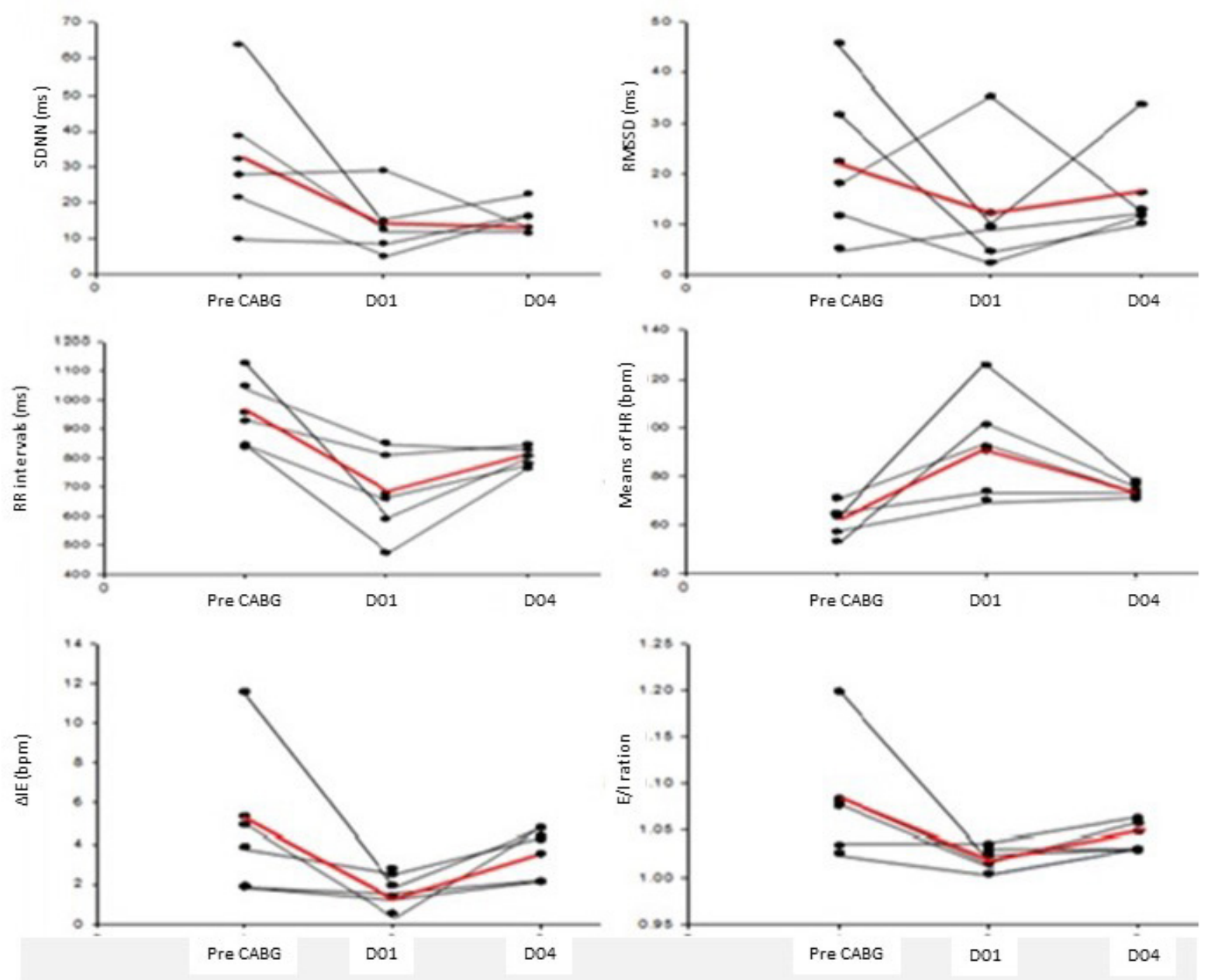

Fig. 1 - Heart rate variability and maneuver of respiratory sinus arrhythmia pre-and post-CABG.

HRV and amplitude of RSA values during M-RSA of the five volunteers. Mean; Patient. $\triangle I E=$ difference between the mean of the highest HR values obtained during the inspiratory phase and the mean of the lowest HR values during the expiratory phase of M-RSA; D01=1st postoperative day; D04=4th postoperative day; E/l ratio=mean of the highest RRi values obtained during the expiratory phase divided by the means of the lowest RRi values of the inspiratory phase of the M-RSA; HR=heart rate; HRV=heart rate variability; M-RSA=maneuver of accentuation of respiratory sinus arrhythmia; $R M S S D=$ square root of the mean square of successive differences between adjacent RRi divided by the number of RRi minus one in $\mathrm{ms}$; RRi=R-R intervals; SDNN=standard deviation of all RRi in $m s$ 
Takeyama et al. (2000) ${ }^{[13]}$ showed that physical exercise during the postoperative period of CABG improves functional capacity and parasympathetic modulation. Therefore, analyzing the autonomic modulation in the postoperative period of CABG would give us the possibility to evaluate the improvement of this system during exercise application, contributing to the standardization of $\mathrm{CP}$ prescription, in addition to improved safety and efficiency ${ }^{[8]}$.

Pulmonary function impairment after CABG is expected and has several causes, such as: sternotomy, pain, pleurotomy by graft incision ${ }^{[14]}$, pleural drainage insertion site ${ }^{[7,14,15]}$, diaphragmatic dysfunction, general anesthesia and reflex dysfunction of the phrenic nerve $e^{[8,16,17]}$, in addition to the use of cardiopulmonary bypass ${ }^{[16,17]}$. Changes in pulmonary mechanics, restrictive respiratory pattern, and shallow breathing are commonly observed in these patients. In this context, a variety of respiratory maneuvers were the basis of $\mathrm{CP}$ care in post-CABG patients. However, early mobilization has proved to be a central strategy in the management of patients undergoing this procedure ${ }^{[18,19]}$.

Data analyzed during M-RSA are unpublished so far in this population. We believe that the improvement in the amplitude of M-RSA is probably related to the fact that these patients, in addition to improving autonomic modulation, also improved pulmonary function with inclusion in CP care. Respiratory exercises and mobilization are able to increase tidal volume, which in turn directly alter the M-RSA amplitude data. The improvement of pulmonary dysfunction provides these patients early cardiopulmonary reconditioning to perform their daily life activities, to generate better prognoses and less possibility of new cardiovascular events $s^{[7,10,16-19]}$.

The limiting conditions of the present study are the sample size, the difficulty in keeping the protocol days unchanged and the lack of daily data, allowing a more precise follow-up of what happened during the entire hospitalization.

\section{CONCLUSION}

In conclusion, it was possible to observe better values of autonomic modulation close to the hospital discharge period, suggesting that the use of an exercise-based cardiovascular physiotherapy protocol may have favored this finding. The indexes that correspond to vagal modulation were the most expressive. The amplitude of M-RSA, described for the first time in this population and during the intervention, also showed values suggestive of improvement close to the $4^{\text {th }}$ postoperative day.

Financial support: The authors would like to thank all the subjects who participated in the research and all team who provided support during data collection. Additionally, we would like to thank the National Council for Scientific and Technological Development (CNPq - protocol: 487375/2012-2) and the Carlos Chagas Filho Foundation for Supporting Research in the State of Rio de Janeiro (FAPERJ - protocol: E-26/110.827/2012) for financial support. Finally, we would like to thank our colleagues from the Research Group on Cardiorespiratory Evaluation and Rehabilitation (GECARE).

\section{No conflict of interest.}

\section{Authors' roles \& responsibilities}

BLS Substantial contributions to the conception or design of the work; or the acquisition, analysis, or interpretation of data for the work; final approval of the version to be published

RRS Acquisition, analysis, or interpretation of data for the work; final approval of the version to be published

HVR Substantial contributions to the conception or design of the work; or the acquisition, analysis, or interpretation of data for the work; final approval of the version to be published

ACAR Acquisition, analysis, or interpretation of data for the work; final approval of the version to be published

PSS Acquisition, analysis, or interpretation of data for the work; final approval of the version to be published

IA Final approval of the version to be published

LF Drafting the work or revising it critically for important intellectual content; agreement to be accountable for all aspects of the work in ensuring that questions related to the accuracy or integrity of any part of the work are appropriately investigated and resolved; final approval of the version to be published

SG Drafting the work or revising it critically for important intellectual content; agreement to be accountable for all aspects of the work in ensuring that questions related to the accuracy or integrity of any part of the work are appropriately investigated and resolved; final approval of the version to be published

MSR Drafting the work or revising it critically for important intellectual content; agreement to be accountable for all aspects of the work in ensuring that questions related to the accuracy or integrity of any part of the work are appropriately investigated and resolved; final approval of the version to be published

\section{REFERENCES}

1. Heart rate variability: standards of measurement, physiological interpretation and clinical use. Task force of the European society of cardiology and the North American society of pacing and electrophysiology. Circulation. 1996;93(5):1043-65.

2. Trimer R, Cabidu R, Sampaio LL, Stirbulov R, Poiares D, Guizilini S, et al. Heart rate variability and cardiorespiratory coupling in obstructive sleep apnea: elderly compared with young. Sleep Med. 2014;15(11):1324-31. doi:10.1016/j.sleep.2014.05.028.

3. Reis MS, Deus AP, Simões RP, Aniceto IA, Catai AM, Borghi-Silva A. Autonomic control of heart rate in patients with chronic cardiorespiratory disease and in healthy participants at rest and during a respiratory sinus arrhythmia maneuver. Rev Bras Fisioter. 2010;14(2):106-13.

4. De Oliveira Grassi D. Estudo da arritmia sinusal respiratória e da variabilidade da frequência cardíaca de homens jovens e de meia idade. Rev Soc Cardiol. 2003;13(3 supl A):15-24.

5. Hayano J, Yasuma F, Okada A, Mukai S, Fujinami T. Respiratory sinus arrhythmia. A phenomenon improving pulmonary gas exchange and circulatory efficiency. Circulation. 1996;94(4):842-7. doi:10.1161/01. cir.94.4.842. 
6. Laitio T, Jalonen J, Kuusela T, Scheinin H. The role of heart rate variability in risk stratification for adverse postoperative cardiac events. Anesth Analg. 2007;105(6):1548-60. doi:10.1213/01.ane.0000287654.49358.3a.

7. Guizilini S, Alves DF, Bolzan DW, Cancio AS, Regenga MM, Moreira RS, et al. Sub-xyphoid pleural drain as a determinant of functional capacity and clinical results after off-pump coronary artery bypass surgery: a randomized clinical trial. Interact Cardiovasc Thorac Surg. 2014;19(3):382-7. doi:10.1093/icvts/ivu138.

8. Andrejaitiene J, Sirvinskas E, Bolys R. The influence of cardiopulmonary bypass on respiratory dysfunction in early postoperative period. Medicina (Kaunas). 2004;40 Suppl 1:7-12.

9. Pantoni CB, Mendes RG, Di Thommazo-Luporini L, Simões RP, AmaralNeto $\mathrm{O}$, Arena $\mathrm{R}$, et al. Recovery of linear and nonlinear heart rate dynamics after coronary artery bypass grafting surgery. Clin Physiol Funct Imaging. 2014;34(6):449-56. doi:10.1111/cpf.12115.

10. Mendes RG, Simões RP, de Souza Melo Costa F, Pantoni CB, Di Thommazo L, Luzzi S, et al. Left-ventricular function and autonomic cardiac adaptations after short-term inpatient cardiac rehabilitation: a prospective clinical trial. J Rehabil Med. 2011;43(8):720-7. doi:10.2340/16501977-0843.

11. Soares PP, Moreno AM, Cravo SL, Nóbrega AC. Coronary artery bypass surgery and longitudinal evaluation of the autonomic cardiovascular function. Crit Care. 2005;9(2):R124-31. doi:10.1186/cc3042.

12. Lee JY, Han S, Ahn JM, Park DW, Kang SJ, Lee SW, et al. Impact of participation in phase I and phase II cardiac rehabilitation on long- term survival after coronary artery bypass graft surgery. Int J Cardiol. 2014;176(3):1429-32. doi:10.1016/j.jijcard.2014.08.041.

13. Takeyama J, Itoh H, Kato M, Koike A, Aoki K, Fu LT, et al. Effects of physical training on the recovery of the autonomic nervous activity during exercise after coronary artery bypass grafting: effects of physical training after CABG. Jpn Circ J. 2000;64(1 1):809-13. doi:10.1253/jcj.64.809.

14. Berrizbeitia LD, Tessler S, Jacobowitz IJ, Kaplan P, Budzilowicz L, Cunningham JN. Effect of sternotomy and coronary bypass surgery on postoperative pulmonary mechanics. Comparison of internal mammary and saphenous vein bypass grafts. Chest. 1989;96(4):873-6. doi:10.1378/ chest.96.4.873.

15. Wiwanitkit V. Post-cardiac surgery anesthesia: comparison of patientcontrolled regimens. Rev Bras Cir Cardiovasc. 2010;25(3):429.

16. Conti VR. Pulmonary injury after cardiopulmonary bypass. Chest. 2001;119(1):2-4. doi:10.1378/chest.119.1.2.

17. Tzani P, Chetta A, Olivieri D. Patient assessment and prevention of pulmonary side-effects in surgery. Curr Opin Anaesthesiol. 2011;24(1):27. doi:10.1097/ACO.0b013e328341abb3.

18. Borghi-Silva A, Mendes RG, Costa Fde S, Di Lorenzo VA, Oliveira CR, Luzzi $S$. The influences of positive end expiratory pressure (PEEP) associated with physiotherapy intervention in phase I cardiac rehabilitation. Clinics (Sao Paulo). 2005;60(6):465-72. doi:10.1590/s1807-59322005000600007.

19. Hirsch JA, Bishop B. Respiratory sinus arrhythmia in humans: how breathing pattern modulates heart rate. Am J Physiol. 1981;241(4):H6209. doi:10.1152/ajpheart.1981.241.4.H620. 